\title{
Esternotomia Parcial Mediana em H. Um Novo Acesso para a Cirurgia Cardíaca
}

\author{
Danton R. Loures, Leonardo A. Mulinari, André L. Tyszka, Edison Ribeiro, Roberto G. Carvalho, \\ Rui Almeida \\ Curitiba, PR
}

\begin{abstract}
Objetivo - Apresentar a nossa experiência com a toracotomia minimamente invasiva (TMI) em pacientes com doença valvar e descrever um novo tipo de toracotomia.

Métodos - Vinte e cinco pacientes com doença valvar, como maior problema cardíaco, foram submetidos a operação em um período de 5 meses. A incisão utilizada foi uma TMI em duas formas, uma em Tinvertido e outra em $H$. Nesta série, as reoperações constituíram $24 \%$.

Resultados - O tamanho da incisão de pele variou de 7,5 a 11,0cm (média =9,1cm). O tempo de permanência médio na UTIfoi de 31 he hospitalar de 4,8 dias. Em todos os pacientes a cirurgia pôde ser realizada apropriadamente através deste acesso, sendo que em apenas um, foi necessária esternotomia total.

Conclusão - A TMI apresenta algumas vantagens, como melhor resultado estético, menos dor e maior estabilidade torácica. Estes fatores possibilitam uma extubação precoce, diminuindo o tempo de internação hospitalar. A esternotomia em H apresenta como grande benefício a exposição da parte mais central do tórax, permitindo um melhor acesso a todas as valvas cardíacas. Esta técnica não apresentou complicações nas reoperações, o que nos faz acreditar que seja tão segura quanto a esternotomia total.
\end{abstract}

Palavras-chave: esternotomia parcial, cirurgia minimamente invasiva, valvopatias

\section{Partial Sternotomy in H.A New Approach for Cardiac Surgery}

Purpose - To present our results with the minimally invasive thoracotomy (MIT) in patients with valve disease and to describe a new type of thoracotomy.

Methods - Twenty-five patients with valve disease as the major cardiac problem were operated during a five month period. Two types of incisions were used: one in an inverted-T fashion and another in a H fashion. Fourteen patients were submitted to an inverted-T incision and eleven to a H incision. In $24 \%$ of the patients reoperation was being performed.

Results - The incisions ranged from 7.5 to $11 \mathrm{~cm}$ (mean $9.1 \mathrm{~cm})$, mean ICU stay was 31 h and hospital stay 4.8 days. In all but one patient the operation could be adequately in performed through the MIT, in only one patient total sternotomy had to be performed.

Conclusion - The MIT may have some advantages such as, better aesthetic results, less pain and more sternal stability. These factors may improve early extubation and decrease hospital stay. The H type incision allows an adequate exposition of the most central part of the chest and all heart valves with adequate exposure. This technique led to no complications during reoperations and we believe it to be as safe as the total sternotomy.

Key-words: sternotomy, minimally invasive surgery, heart valve surgery

Arq Bras Cardiol, volume 70 (n 2), 71-73, 1998

A cirurgia cardiovascular tem se destacado como uma especialidade médica que, constantemente, vem apresentando inovações técnicas e evoluções nas alternativas de tratamento. Aos pacientes, ofertam-se diversos procedi-

Hospital de Clínicas da Universidade Federal do Paraná - Curitiba Correspondência: Danton R. Rocha Loures - Rua Augusto Stelfeld, 2088 - 80730150 - Curitiba, PR

Recebido para publicação em 22/9/97

Aceito em 16/10/97 mentos terapêuticos dentro da cardiologia clínica, invasiva ou na cirurgia cardiovascular, podendo se optar, com segurança, na dependência direta de melhores resultados. Nestes últimos dois anos, muitas equipes de cirurgia cardiovascular introduziram a toracotomia minimamente invasiva, como acesso à correção de diversos tipos de cardiopatias. Os resultados apresentados mostraram a redução no tamanho da incisão de pele de $25 \mathrm{~cm}$ para menos de $10 \mathrm{~cm}$, uma menor agressão cirúrgica, menor sangramento no pós-ope- 
ratório (PO), menor tempo de permanência na UTI e de internação hospitalar, acarretando uma diminuição dos custos. Tudo isto sem colocar em risco a segurança do paciente e com resultados semelhantes à toracotomia convencional. As maiores críticas a esta nova abordagem tem sido a diversidade de tipos de toracotomias (ântero-lateral direita e esquerda, mediana superior, inferior, etc.) e suas limitações nas indicações cirúrgicas. Os autores apresentam sua experiência inicial com a toracotomia minimamente invasiva e introduzem um acesso inédito denominado de esternotomia parcial mediana em $\mathrm{H}$, que possibilitou o tratamento cirúrgico de todos os tipos de valvopatias, sendo elas isoladas, associadas ou, mesmo, nas reoperações.

\section{Métodos}

Estudamos, prospectivamente, todos os pacientes com doenças valvares operados, no Hospital de Clínicas da UFP, através de uma toracotomia minimamente invasiva, no período de novembro/96 a março/97. Foram submetidos a este acesso 25 pacientes, com idade média de 37,75 (16 a 57) anos, sendo 13 do sexo feminino e 12 do masculino.

Esternotomia parcial em T invertido - Nos primeiros 14 pacientes, após uma incisão mediana de pele de cerca de $9 \mathrm{~cm}$, realizou-se uma esternotomia superior em Tinvertido, iniciada na fúrcula esternal e estendendo-se, longitudinalmente, através do manúbrio e corpo até altura do $3^{\circ}$ espaço intercostal, quando então, o esterno era seccionado transversalmente.

Devida a uma inadequada exposição do campo cirúrgico, para se realizar uma $2^{\mathrm{a}}$ retroca de prótese mitral, onde houve a necessidade de uma conversão para técnica tradicional, ou seja, para uma esternotomia total, nas operações subseqüentes, realizamos uma modificação técnica, como se segue:

Esternotomia parcial mediana em $\mathrm{H}$ - Através de uma incisão de pele semelhante à anterior, com cerca de $9 \mathrm{~cm}$ de comprimento, o esterno foi abordado e seccionado em dois níveis diferentes: o $1^{\circ}$, no manúbrio esternal, abaixo da inserção da $1^{\mathrm{a}}$ costela, e o $2^{\circ}$, na altura do $4^{\circ}$ espaço intercostal. Finalmente o esterno era seccionado, longitudinalmente, entre as duas incisões transversais (esternotomia em $\mathrm{H}$ ) (fig. 1).

Nos casos de tratamento cirúrgico da valva mitral, sua abordagem foi feita através do teto do átrio esquerdo, entre a veia cava superior e a aorta. O restante da operação foi transcorrida de maneira habitual, de acordo com a rotina do serviço.

\section{Resultados}

Neste período, realizamos um total de 25 operações, em que em 19 casos foram realizados procedimentos isolados e, em seis procedimentos múltiplos, (tab. I). O tratamento cirúrgico da valva mitral foi o mais freqüente, correspondendo a 11 casos (comissurotomia mitral (7) e troca da valva (4)). A valva aórtica foi substituída em sete casos e,

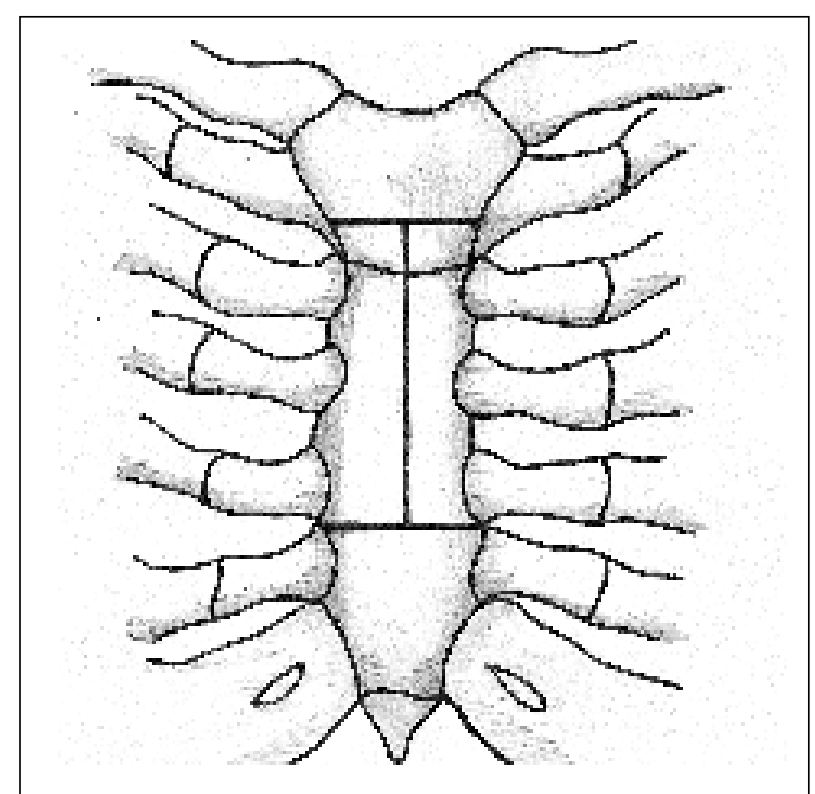

Fig. 1 - Ilustração da esternotomia em H.

em um, foi realizada apenas a toilette da prótese, tornandoa em condições ideais para a sua preservação. Os procedimentos associados consistiram de: dupla troca aórtica e mitral (3), troca da valva aórtica e plastia da valva mitral implante de anel de Carpentier - (1), troca da valva mitral e plastia da valva tricúspide - (1) e troca da valva aórtica e revascularização miocárdica - enxerto venoso para o ramo diagonal e artéria torácica interna para o ramo interventricular anterior - (1). Dos 25 casos, 19 (76\%) eram primeira operação e seis (24\%) eram reoperações, sendo que em três $(12 \%)$ eram $2^{\text {a }}$ reoperação.

O tamanho da incisão de pele variou de 7,5 a $11 \mathrm{~cm}$, com uma média de $9,1 \mathrm{~cm}$. Com esta técnica, o tempo de circulação extracorpórea e o tempo de pinçamento aórtico foram semelhantes às técnicas tradicionais, inclusive nas reoperações.

O volume de drenagem torácica médio foi de $327 \mathrm{ml}$ (50 a 1820), sendo que o paciente que apresentou a maior drenagem necessitou ser reexplorado.

O tempo de permanência na UTI variou de $16 \mathrm{~h}$ a seis dias, com uma média de $31 \mathrm{~h}$, sendo que 11 (44\%) pacientes permaneceram menos de $24 \mathrm{~h}$ sob cuidados intensivos. $\mathrm{O}$ tempo de internação hospitalar no PO foi de 4,8 dias ( 3 a 19) e $12(48 \%)$ pacientes receberam alta hospitalar até o $4^{\circ}$ dia.

As complicações operatórias (até 30 dias) foram: bloqueio atrioventricular total com implante de marcapasso definitivo em um caso, sangramento excessivo necessitando de reexploração em um, assistência ventilatória prolongada (cinco dias) em um paciente. Devida a dificuldade de acesso pela esternotomia parcial superior, em um caso de $2^{\mathrm{a}}$ reoperação para troca da valva mitral houve a necessidade de conversão para técnica tradicional (esternotomia total), e a partir daí, iniciamos a técnica de esternotomia parcial em $\mathrm{H}$ para os próximos casos. Um paciente que necessitou de reoperação por sangramento, em que se evidenciou dissec- 


\begin{tabular}{|lccc|}
\hline \multicolumn{4}{|c|}{ Tabela I - Tipo de procedimentos realizados } \\
\hline Procedimentos isolados & $\mathrm{n}=19$ & $\mathrm{~T}$ & $\mathrm{H}$ \\
\hline Comissurotomia mitral & 7 & 6 & 1 \\
Troca de valva mitral (VMi) & 3 & 2 & 1 \\
Retroca de prótese mitral & 1 & $1 *$ & - \\
Troca de valva aórtica (VAo) & 6 & 3 & 3 \\
Re-retroca de prótese aórtica & 1 & 1 & - \\
"Toilette" de prótese aórtica & 1 & 1 & - \\
\hline Procedimentos associados & $\mathrm{n}=6$ & $\mathrm{~T}$ & $\mathrm{H}$ \\
\hline Retroca VMi e Vao & 3 & 1 & 2 \\
Troca VAo + plastia Vmi & 1 & - & 1 \\
Troca VMi + plastia V. tricúspide & 1 & - & 1 \\
Troca VAo + revasc. Miocárdica & 1 & - & 1 \\
\hline T- esternotomia parcial mediana em T invertido; H- esternotomia parcial \\
mediana em H; *- caso de esternotomia em T que foi convertida para total. \\
\hline
\end{tabular}

ção aórtica, apresentou grave comprometimento cerebral e faleceu no $2^{\circ}$ dia de $P O$.

\section{Discussão}

Atualmente, em todas as áreas cirúrgicas, tem se buscado o emprego de técnicas menos agressivas, visando maior conforto para o paciente e uma mais rápida recuperação. Assim, na cirurgia cardíaca, já vem se tornando rotineiro o emprego de técnicas menos invasivas para a revascularização miocárdica e a correção de cardiopatias congênitas. Apresentamos, nossa experiência com o uso da toracotomia minimamente invasiva em pacientes com doenças valvares e a ampla aplicabilidade da esternotomia parci- al em $\mathrm{H}$, como novo acesso para o tratamento cirúrgico de cardiopatias.

Por permitir uma abordagem mais central, esta nova técnica traz a vantagem de permitir o acesso mais amplo ao coração, além de oferecer maior facilidade na abordagem da valva mitral nas reoperações, tendo sido ampliada sua indicação para os pacientes com doenças associadas, como a doença da valva tricúspide, por possibilitar o cadarceamento de ambas veias cavas e a revascularização miocárdica, facilitar a dissecção da artéria mamária e o acesso aos diversos sistemas coronários, além de manter as indicações para a valva aórtica. Esta técnica também conserva a estabilidade da caixa torácica em duas porções: na porção superior, através do manúbrio esternal articulado com a clavícula e a $1^{\mathrm{a}}$ costela, e da sua porção inferior, traduzida pela articulação entre a $5^{\mathrm{a}} \mathrm{e} 6^{\mathrm{a}}$ costelas e o corpo esternal.

Devida à restrição imposta pelas áreas mantidas fixas, 0 acesso impede uma abertura exagerada do afastador de tórax, diminuindo a dor no PO, obtendo um melhor desempenho respiratório no $\mathrm{PO}$ imediato, facilitando a extubação e a deambulação e permitindo uma alta precoce com segurança.

Com esta nossa casuística inicial, pudemos observar que a toracotomia minimamente invasiva apresenta vantagens, como menor incisão de pele com melhores resultados estéticos, maior estabilidade torácica, menos dor no PO, menor tempo de permanência em UTI e hospitalare, conseqüentemente, diminuição nos custos, não acrescentando riscos de morbimortalidade aos pacientes. Agora com a esternotomia parcial em $\mathrm{H}$ adiciona-se às vantagens citadas, a possibilidade de, através da mesma incisão, corrigir-se a grande maioria das doenças valvares isoladas ou associadas, sendo que a técnica, se aplica aos casos de reoperações.

\section{Referências}

1. Grandjean JG, Mariani AM, Ebels T - Coronary reoperation via small laparotomy using right gastroepiploic artery without CPB. Ann Thorac Surg 1996; 61: 1853-5.

2. Lytle BW - Editorial: Minimally invasive cardiac surgery. J Thorac Cardiovasc Surg 1996; 111: 586

3. Schwartz DS, Ribakove GH et al - Minimally invasive cardiopulmonary bypass with cardioplegic arrest: a closed chest technique with equivalent myocardial protection. J Thorac Cardiovasc Surg 1996; 111: 557-66.

4. Chitwood Jr WR, Elbeery JR et al - Video-assisted minimally invasive mitral valve surgery: The "micro-mitral" operation. J Thorac Cardiovasc Surg 1996; 111: 413-14.

5. Stanbridge RDL, Symons GV et al - Minimal-access surgery for coronary artery revascularization. Lancet 1995; 23: 837.

6. Stevens JH, Burdon TA et al - Port-access coronary artery bypass with cadioplegic arrest: Acute and chronic canine studies. Ann Thorac Surg 1996; 62: 435-41.

7. Cosgrove DM, Sabik JF - Minimally invasive approach for aortic valve operations. Ann Thoracic Surg 1996; 62: 596-7.

8. Tribble CG, Killinger Jr WA et al - Anterolateral thoracotomy as alternative to repeat median sternotomy for replacement of the mitral valve. Ann Thorac Surg 1995; 59: 255-6.

9. Hirose H, Umeda S et al - Another approach for aortic valve replacement through left thoracotomy. Ann Thorac Surg 1994; 58: 884-6.

10. Dalton ML, Connaly SR, Sealy WC - Julian's reintroduction of milton's operation. Ann Thorac Surg 1992; 53: 532-3.

11. Wilson WR, Ilbawi MN et al - Partial median sternotomy for repair of heart defects: A cosmetic approach. Ann Thorac Surg 1992; 54: 892-3.

12. Tatebe $\mathrm{S}$, Eguchi $\mathrm{S}$ et al - Limited vertical skin incision for median sternotomy Ann Thorac Surg 1992; 54: 787-8.

13. Uppal R, Wolfe WG et al - Right thoracotomy for reoperative right coronary artery bypass procedures. Ann Thorac Surg 1994; 54: 123-5.

14. Pompili MF, Stevens JH et al - Port-access mitral valve replacement in dogs. J Thorac Cardiovasc Surg 1996;112: 1268-74.

15. Calafiori AM, Di Giammarco G et al - Left anterior descending coronary artery grafting via left anterior small thoracotomy without cardiopulmonary bypass. Ann Thorac Surg 1996; 61: 1658-65.

16. Acuff TE, Landreneau RJ et al - Minimally invasive coronary artery bypass grafting. Ann Thorac Surg 1996; 61: 135-7. 